Open Access

\title{
Increased glucocorticoid receptor expression in sepsis is related to heat shock proteins, cytokines, and cortisol and is associated with increased mortality
}

Konstantinos Vardas ${ }^{1,2}$, Stavroula $l l i{ }^{2}$, Amalia Sertedaki ${ }^{3}$, Evangelia Charmandari ${ }^{3}$, Efrossini Briassouli ${ }^{4}$, Dimitris Goukos ${ }^{4}$, Kleovoulos Apostolou' ${ }^{1}$ Katerina Psarra ${ }^{5}$, Efthimia Botoula ${ }^{6}$, Stylianos Tsagarakis ${ }^{6}$, Eleni Magira ${ }^{1}$, Christina Routsi ${ }^{1}$, Constantine A. Stratakis ${ }^{7}$, Serafim Nanas ${ }^{1}$ and George Briassoulis ${ }^{2^{*}}$ (D)

* Correspondence: ggbriass@otenet.gr 2Pediatric Intensive Care Unit, University Hospital, University of Crete, 71500 Heraklion, Crete, Greece

Full list of author information is available at the end of the article

\begin{abstract}
Background: The purposes of this study are to examine if the human glucocorticoid receptor (hGR) isoform-a mRNA and hGR protein expressions are deficient in the acute phase of sepsis (S) compared to systemic inflammatory response syndrome (SIRS) and healthy subjects $(\mathrm{H})$ and to evaluate if the $\mathrm{hGRa}$ and $\mathrm{hGR}$ alterations are associated with cortisol changes and if they are related to (1) extracellular and intracellular heat shock proteins (HSP) 72 and 90a; (2) ACTH, prolactin, and interleukins (ILs); and (3) outcome.

Methods: Patients consecutively admitted to a university hospital intensive care unit (ICU) with S $(n=48)$ or SIRS $(n=40)$ were enrolled in the study. Thirty-five H were also included. Total mRNA was isolated from peripheral blood samples and cDNA was prepared. RT-PCR was performed. Intracellular hGR and HSP expression in monocytes and/or neutrophils was evaluated using four-colour flow cytometry. Serum prolactin, $\mathrm{ACTH}$, and cortisol concentrations were also measured. ELISA was used to evaluate serum ILs and extracellular (e) HSPs (eHSP72, eHSP90a).
\end{abstract}

Results: $h$ GR protein was higher in $\mathrm{S}$ compared to $\mathrm{H}$ and SIRS; hGRa mRNA was higher in $\mathrm{S}$ compared to $\mathrm{H}(p<0.05)$. In sepsis, hGR protein and eHSP72 were higher among non-survivors compared to survivors $(p<0.05)$. The hGR MFI and hGRa mRNA fold changes were significantly related to each other $\left(r_{s}=0.64, p<0.001\right)$. Monocyte hGR protein expression was positively correlated with extracellular and intracellular HSPs, cortisol, and ILs and negatively to organ dysfunction $(p<0.05)$. HSPs, hGR, and cortisol were able to discriminate sepsis from SIRS (AUROC $>0.85, p<0.05$ ). In sepsis, monocyte-hGR protein and eHSP72 were strong predictors of mortality (AUROC $>0.95, p<0.04$ ).

Conclusions: Acute-phase sepsis is associated with increased hGR expression and cortisol concentrations, possibly implying no need for exogenous steroids. At this stage, hGR is able to predict sepsis and outcome and is related to stress-activated bio-molecules and organ dysfunction.

Keywords: Glucocorticoid receptor (GR), Cortisol, Heat shock protein 72, HSP90a, Sepsis, SIRS 


\section{Background}

Sepsis is a life-threatening organ dysfunction caused by a dysregulated host response to infection [1]. The activation of the hypothalamic-pituitary-adrenal (HPA) axis results in increased cortisol concentrations driven by increased secretion of ACTH [2]. Also, decreased activity of the cortisol metabolizing enzymes impairs cortisol clearance, enhances hypercortisolism, and suppresses ACTH secretion by feedback inhibition [3].

In humans, glucocorticoids (GCs) exert their effects through binding to their cognate receptor, the glucocorticoid receptor, a ligand-dependent transcription factor [4]. The human glucocorticoid receptor (hGR) is encoded by the NR3C1 gene, which is located on the long arm of chromosome 5 (5q31.3) and is composed of ten exons. Alternative splicing of exon nine generates two highly homologous receptor isoforms, hGR $\alpha$, and hGR $\beta$. The residing in the cytoplasm hGR $\alpha$ functions as a ligand-dependent transcription factor [4]. The hGR $\beta$ does not bind glucocorticoid agonists and exerts a dominant negative effect on the transcriptional activity of hGR $\alpha$ [5].

In the absence of ligand, the hGR $\alpha$ resides mostly in the cytoplasm of cells as part of a hetero-oligomeric complex, which contains chaperone heat shock proteins (HSPs) 90 and 70. HSP90 regulates ligand binding, as well as cytoplasmic retention of hGR $\alpha$ by exposing the ligand-binding site and masking the two nuclear localization sequences [4]. HSP90 $\alpha$ and HSP72 work together as the only two essential components of the five-protein system for allowing $h G R \alpha$ to bind the incoming steroid hormone by enhancing its affinity for the ligand [6]. Upon ligand binding and phosphorylation, the hGR $\alpha$ dissociates from HSPs and translocates into the nucleus. Nuclear hGR $\alpha$ binds to glucocorticoid response elements (GREs) of target genes and upregulates or downregulates their expression, depending on GRE sequence and promoter context [4] (Fig. 1).

GCs downregulate the expression of their receptor through transcriptional, posttranscriptional, and posttranslational mechanisms [7]. Thus, treatment with GCs activates a negative feedback loop by inducing a specific critical suppressor of NR3C1 gene, the microRNA-124, thereby impeding the hGR $\alpha$ upregulation and shifting the hGR $\alpha$ hGR $\beta$ ratio toward hGR $\beta$. Such a shift limits or negatively impacts the GC anti-inflammatory effects by aggravating the GC resistance [8]. Septic serum induces the expression of not only hGR $\alpha$ but also of hGR $\beta$ in both T and $B$ cells in culture, supporting a possible GC resistance of specific cell subpopulations in sepsis [9]. In pediatric sepsis, however, hGR $\beta$ mRNA levels did not change significantly during sepsis, verifying inconsistency with results of hGR $\beta$ [10]. By investigating $h G R \alpha$ mRNA expression rather than protein levels [8], these studies produced inconsistent results about the hGR $\alpha$ expression and function in critical illness.

To the best of our knowledge, the assessment of both protein and mRNA hGR $\alpha$ expression in sepsis and systemic inflammatory response syndrome (SIRS) in relation to their hetero-oligomeric HSPs parts, and the stress-induced inflammatory response has not been investigated previously. In this study, we assessed the hGR protein expression in monocytes and the hGR $\alpha$ mRNA in whole blood in intensive care unit (ICU) patients with early-onset sepsis (S) compared to SIRS or healthy 


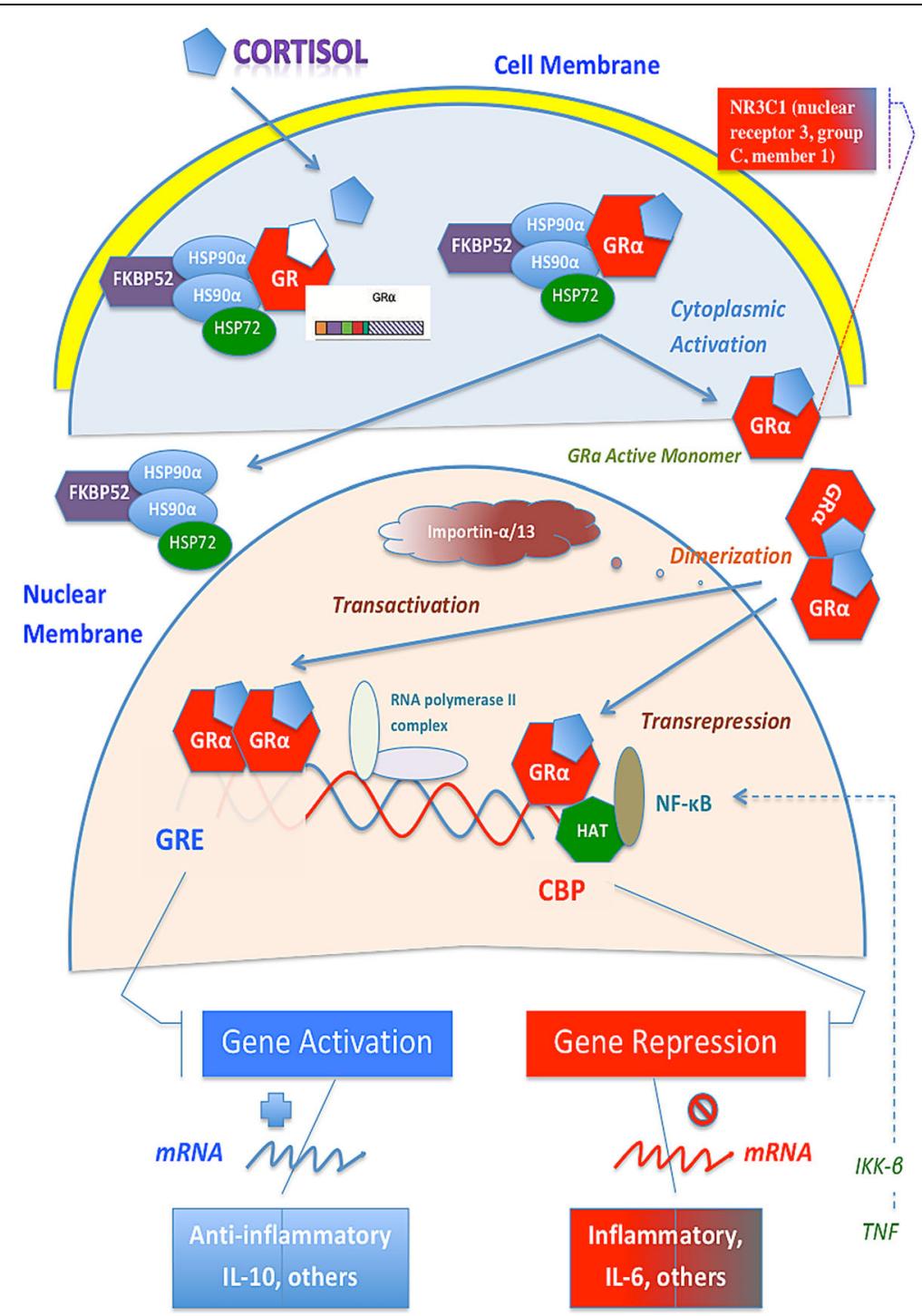

Fig. 1 Glucocorticoids (GC) diffuse across the cell membrane and bind to human glucocorticoid receptor (hGR) in the cytoplasm. In a heat shock protein (HSP) heterocomplex, hGRa is activated (upon ligand binding), is released from HSP72 and HSP90a, and rapidly translocates into the nucleus, where the transcription of target genes is initiated. Through transactivation, binding of two hGRa molecules together as a homodimer to glucocorticosteroid response elements (GRE) in the promoter region of steroid-sensitive genes leads to the transcription of genes encoding anti-inflammatory mediators (i.e., IL-10) and the inhibition of nuclear factor-KB (NF-KB). Through transrepression, the hGRa-GC complex interacts with the activated by NF-KB and other pro-inflammatory transcription factors with intrinsic histone acetyltransferase (HAT) activity switching off multiple activated inflammatory genes (i.e., IL-6). CBP CAMP response element binding protein, IKK $\beta$ inhibitor of I-KB kinase- $\beta$

control subjects $(\mathrm{H})$. We examined the hypothesis that the hormonal-innate immune stress-responding pairs of hGR-cortisol and hGR-HSPs are adequately expressed in the acute phase of sepsis. We also examined the hypothesis that the hGR mRNA fold change and protein expression alterations are associated with simultaneous cortisol, extracellular or intracellular HSP72 or HSP90 $\alpha$ changes, or with stress-activated bio-molecules and organ dysfunction. Finally, we evaluated the ability of the hGR alterations to predict sepsis or outcome. 


\section{Methods}

\section{Patients}

The study was approved by the institutional review board of Evangelismos Hospital and was performed during a 24-month period between September 2013 and September 2015. Written informed consent was obtained from the relatives of patients admitted to the ICU. Patients aged 18-75 years consecutively admitted with early-onset $(<24 \mathrm{~h})$ sepsis/septic shock or SIRS were eligible for enrollment. The sepsis group $(S)$ included patients $(n=48)$ with an identified source of infection and Sequential Organ Failure Assessment (SOFA) [11] score $>2$, according to the updated Sepsis-3 definition [12]. Of those, 59.5\% fulfilled the septic shock criteria (need for inotropic support to maintain MAP>65 $\mathrm{mmHg}$ and lactate levels $>2 \mathrm{mmol} / \mathrm{L})$. The non-infectious SIRS group included trauma patients $(n=40)$ who met at least two of the four conventional criteria for SIRS [13] and represented the first control group (ICU control). The H group included healthy volunteers $(n=35)$, representing the second control group (healthy individuals). Exclusion criteria were (a) malignancy, (b) autoimmune diseases, (c) prior use of corticoids, (d) immune deficiency disorders, and (e) late sepsis or SIRS > $48 \mathrm{~h}$ after admission. Acute Physiology and Chronic Evaluation-II (APACHE II) [14], multiple organ dysfunction (MODS) [15], Simplified Acute Physiology Score-III (SAPS III) [16], and SOFA scores were recorded on admission. Blood samples were obtained at 8 am because of a peripheral CLOCKmediated circadian acetylation of the human GR counter-regulating the actions of diurnally fluctuating cortisol [17]. Blood gas values and clinical laboratory data were obtained from laboratory records.

\section{Laboratory assays \\ RNA isolation, reverse transcription and RT-PCR for GRa (NR3C1)}

Total RNA was isolated from peripheral blood employing the TRIzol Reagent (Ambion by Life Technologies) according to the manufacturer's instructions. RNA (1 $\mu \mathrm{g}$ ) was DNase treated using RQ1 RNase-free DNase (Promega) and reverse transcription was carried employing the Transcriptor First Strand cDNA Synthesis Kit (Roche Applied Science). Duplicate samples of the cDNA underwent employing primers and TaqMan probes designed by TIB MOLBIOL and the LightCycler 480 Probes Master Kit (Roche) on a LightCycler 480 System (Roche). The reference gene used in this study was the RPLP0. Repeated trials for hGR $\beta$ detection in samples of all groups were mostly unsuccessful, so that only hGR $\alpha$ mRNA was measured. Standard curves were constructed using triplicate samples of a standard RNA sample for hGR $\alpha$ and RPLP0. The sequence of the primers and probes of the target and the reference gene were:

hNR3C1a S 5'-TATgCATgAAgTggTTgAAAATCTCC-3'(exon 9)

hNR3C1a A 5'-ggTATCTgATTggTgATgATTTCAgC-3' (exon 9)

hNR3C1 FAM-CATCTCggggAATTCAATACTCATggTCTT-BBQ (exon 9)

RPLP0-F 5'-CTCTGGAGAAACTGCTGCCTCATA-3', (exon 4)

RPLP0-R 5'-GACTTCACATGGGGCAATGG-3', (exon 5)

RPLP0 FAM-AGGACCTCACTGAGATCAGGGACATGT-BBQ (exon 4-5) 
Data analysis was performed using the Advanced Relative Quantification software of the LightCycler 480.

\section{Flow cytometry for human GR, HSP72, and HSP90a}

The monocytes and neutrophils protein expression was evaluated by flow cytometry. Ethylenediaminetetraacetic acid (EDTA)-anticoagulated blood $(100 \mu \mathrm{L})$ was used for flow cytometric analysis of fresh peripheral blood monocyte (m) and neutrophil (n) HSP72 and HSP90 $\alpha$, and monocytes hGR $\alpha$ protein expressed as mean fluorescence intensity (MFI) were determined after staining surface antigens CD33 and CD45 with $5 \mu \mathrm{L}$ monoclonal antibodies CD33-PE/Cy5 and CD45 PE/Cy7 (BioLegend, San Diego, USA) followed by HSP72, HSP90 $\alpha$, and hGR intracellular staining with $5 \mu \mathrm{L}$ HSP72-FITC, $5 \mu \mathrm{L}$ HSP90 $\alpha$ $\mathrm{PE}$, or $10 \mu \mathrm{L}$ anti-hGR-FITC monoclonal antibodies, respectively (Enzo Life Sciences, Ann Arbor, MI, USA). Anti-hGR $\alpha$ antibodies conjugated to any fluorescence were not commercially available. Additionally, although hGR antibody detects all protein isoforms, hGR $\alpha$ is represented in almost $99 \%$ of hGR protein [10]. Non-detection of hGR $\beta$ protein with flow cytometry would also be expected when it couldn't be detected on RNA level. Isotype controls were used to check if blocking was needed. Assays were performed according to the manufacturer's instructions using Flow Cytometer FC-500 (Beckman Coulter, Miami, FL, USA).

\section{ACTH cortisol and prolactin}

During the first $48 \mathrm{~h}$ after admission to ICU, blood samples for determination of ACTH cortisol and prolactin were collected at 08:00 h. Plasma ACTH was measured using the Immulite 2000 Immunoassay Analyzer (Siemens Healthcare Diagnostics, Tarrytown, NY, USA), while serum cortisol and prolactin concentrations were determined using the ADVIA Centaur Immunoassay Analyzer (Siemens Healthcare Diagnostics, Tarrytown, NY, USA).

\section{Cytokines and extracellular heat shock proteins}

Serum cytokine concentrations of IL-6, IL-10, IL-17, and interferon (IFN)- $\gamma$ were measured by ELISA according to the instructions of the manufacturer. Extracellular plasma levels of HSPs (eHSP72 and eHSP90 $\alpha$ ) were analyzed by ELISA assay according to the manufacturers' instructions (Invitrogen Carlsbad, CA, USA and Enzo Life Sciences, Ann Arbor, MI, USA, respectively). Inter- and intra-assay confidence intervals (CI) for each analyte were: IL-6:6.2, 7.8; IL-10:3.25, 2.75 IL-17:3.7 and in process, IFN- $\gamma: 3.5,7.3$, HSP72:7.1, 15.2, HSP90 $\alpha<10$. Sensitivities of assays: IL-6 $<2 \mathrm{pg} / \mathrm{mL}, \mathrm{IL}-10<1 \mathrm{pg} / \mathrm{mL}$, $\mathrm{IL}-17=2 \mathrm{pg} / \mathrm{mL}, \mathrm{IFN}-\gamma=0.03 \mathrm{IU} / \mathrm{mL}, \mathrm{HSP} 72=90 \mathrm{pg} / \mathrm{mL}$, and HSP90 $\alpha=50 \mathrm{pg} / \mathrm{mL}$.

\section{Statistical analysis}

The one-sample Kolmogorov-Smirnoff test was used to determine the data distribution from measured variables. Data are expressed as median values with range for continuous parameters and as frequencies for categorical variables. The Kruskal-Wallis independent samples test was used to perform comparisons among groups, as appropriate. We ran multiple comparison analyses using post hoc Dunn's pairwise tests with Bonferroni corrections for the Kruskal-Wallis $H$ test; the $x^{2}$ test statistic $\left(H^{2}\right)$ was adopted to calculate the effect size $\left(w^{2}=H^{2} /(n-1)\right)$ to avoid the inflation of overall type I error. Between-group comparisons were conducted using the $\chi^{2}$ test for categorical parameters and Spearman's rank correlation coefficient for correlation 
between two continuous variables. The effect size for $x^{2}$ test was measured using the Cramer's $V\left(V=\sqrt{ } x^{2} / n . \mathrm{df}\right)$ goodness of fit. To evaluate predictive values, we calculated the areas under the receiver operating characteristic curves (AUROC) for variables significantly differing between $\mathrm{S}$ and SIRS groups or between survival and nonsurvival sub-cohorts for the sepsis group. For the predictive values of sepsis or mortality we used biomarkers significantly related to them in bivariate correlations, as appropriate. We consider an AUROC $>0.80$ as clinically relevant whereas the optimum cutoff value was calculated by the highest sensitivity and specificity combined (Youden index approach). The positive and negative predictive values were also calculated. A two-sided significance level of 0.05 was used for statistical inference. All statistical analyses were performed using the IBM SPSS Statistics (version 22.0; Chicago, IL).

\section{Results}

\section{Group differences}

Monocytes hGR protein expression was higher in $S$ compared to $H$ and SIRS (Kruskal-Wallis Test, pairwise comparisons); hGR $\alpha$ mRNA was higher in $S$ compared to $\mathrm{H}$ (Table 1). Groups also differed regarding severity of illness, hormonal, inflammatory, intracellular, and extracellular innate immune response. The proportions of variability (effect size) in the ranked dependent variables accounted for by grouping variables were $>0.20$ (medium per Cohen), indicating a fairly strong relationship between sepsis and the expression levels of hGR protein, cortisol, extracellular HSP72 and 90 $\alpha$, SAPSIII, IL-6, procalcitonin (PCT), age, sex, and mortality (Table 1).

None of the patients had received steroids the last month before admission (exclusion criteria). Fifteen $\mathrm{S}$ patients had been started on a stress-dose of hydrocortisone before blood sampling because of catecholamine resistant shock (31\%). Neither hGR mRNA nor MFI differed between the steroid and non-steroid group. Although HSP90 $\alpha$ did not differ between groups, eHSP72 was higher and nHSP72 was lower in the group receiving hydrocortisone (stress dose). Prolactin concentrations were higher but ACTH were lower in females compared to males ( $p<0.001)$. Cortisol, hGR, hGR $\alpha$ mRNA, HSPs, and ILs did not differ between males and females.

\section{Monocytes hGR bivariate correlations}

The hGR MFI and hGR $\alpha$ mRNA fold change were significantly related to each other $\left(r_{\mathrm{s}}=0.64, p<0.001\right)$, even when the hGR protein expression was corrected by the percentages of WBC monocytes $\left(r_{\mathrm{s}}=0.59, p<0.01\right)$. The expression of hGR protein in monocytes was related to eHSP90 $\alpha\left(r_{\mathrm{s}}=0.33, p<0.05\right)$, eHSP72 $\left(r_{\mathrm{s}}=0.41\right.$, $p<0.02), \operatorname{mHSP} 90 \alpha\left(r_{\mathrm{s}}=0.60, p<0.001\right), \operatorname{nHSP90} \alpha\left(r_{\mathrm{s}}=0.56, p<0.001\right), \mathrm{mHSP72}$ $\left(r_{\mathrm{s}}=0.41, p<0.02\right)$, cortisol $\left(r_{\mathrm{s}}=0.38, p<0.03\right)$, IL-6 $\left(r_{\mathrm{s}}=0.50, p<0.003\right)$, and IL$10\left(r_{\mathrm{s}}=0.40, p<0.025\right)$ (Fig. $\left.2 \mathrm{a}-\mathrm{j}\right)$. hGR MFI was also negatively related to $\mathrm{PO}_{2}$ $\left(r_{s}=-0.72, p<0.02\right), \mathrm{PO}_{2} / \mathrm{FiO}_{2}\left(r_{\mathrm{s}}=-0.76, p<0.005\right)$, and albumin $\left(r_{\mathrm{s}}=-0.77, p<0.01\right)$ and positively to MODS $\left(r_{\mathrm{s}}=0.61, p<0.01\right)$ and SOFA $\left(r_{\mathrm{s}}=0.56, p<0.03\right)$. hGR $\alpha$ mRNA was related to eHSP72 $\left(r_{\mathrm{s}}=0.34, p<0.02\right)$, ACTH $\left(r_{\mathrm{s}}=0.36, p<0.005\right)$, IFN$\gamma\left(r_{\mathrm{s}}=0.42, p=0.001\right)$, C-reactive protein (CRP) $\left(r_{\mathrm{s}}=0.25, p<0.05\right)$, and WBC $\left(r_{\mathrm{s}}=0.38\right.$, $p<0.002)$. No significant associations were found between hGR $\alpha$ and APACHE II, SAPS III, temperature, PCT, IL-17, lactate, LDH, or glucose. 


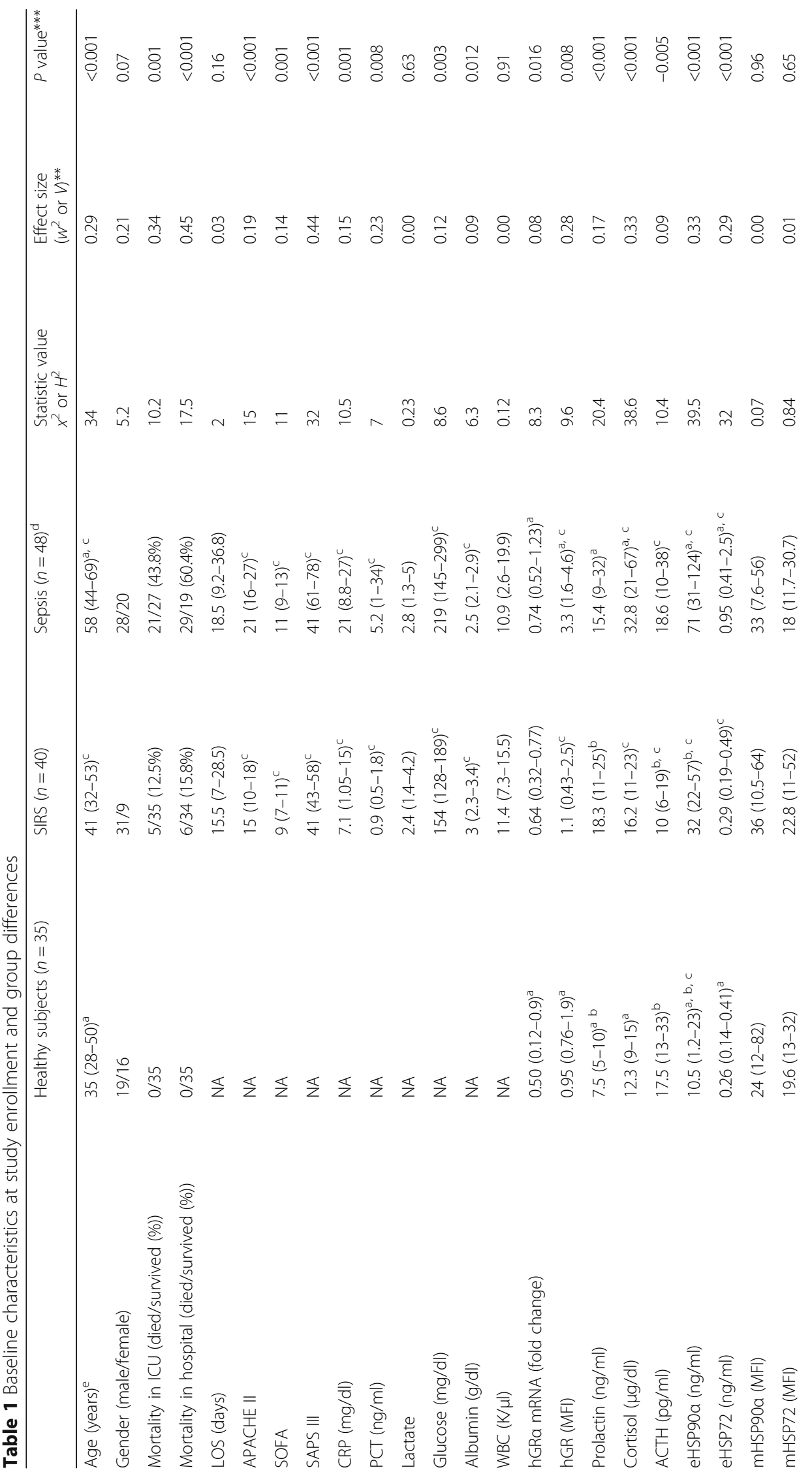




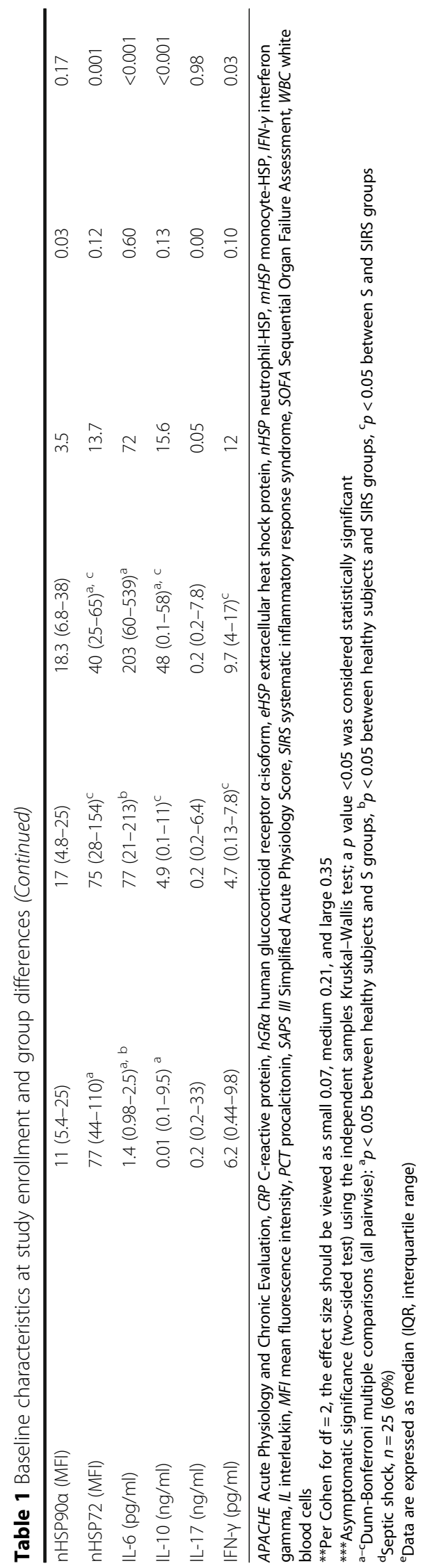




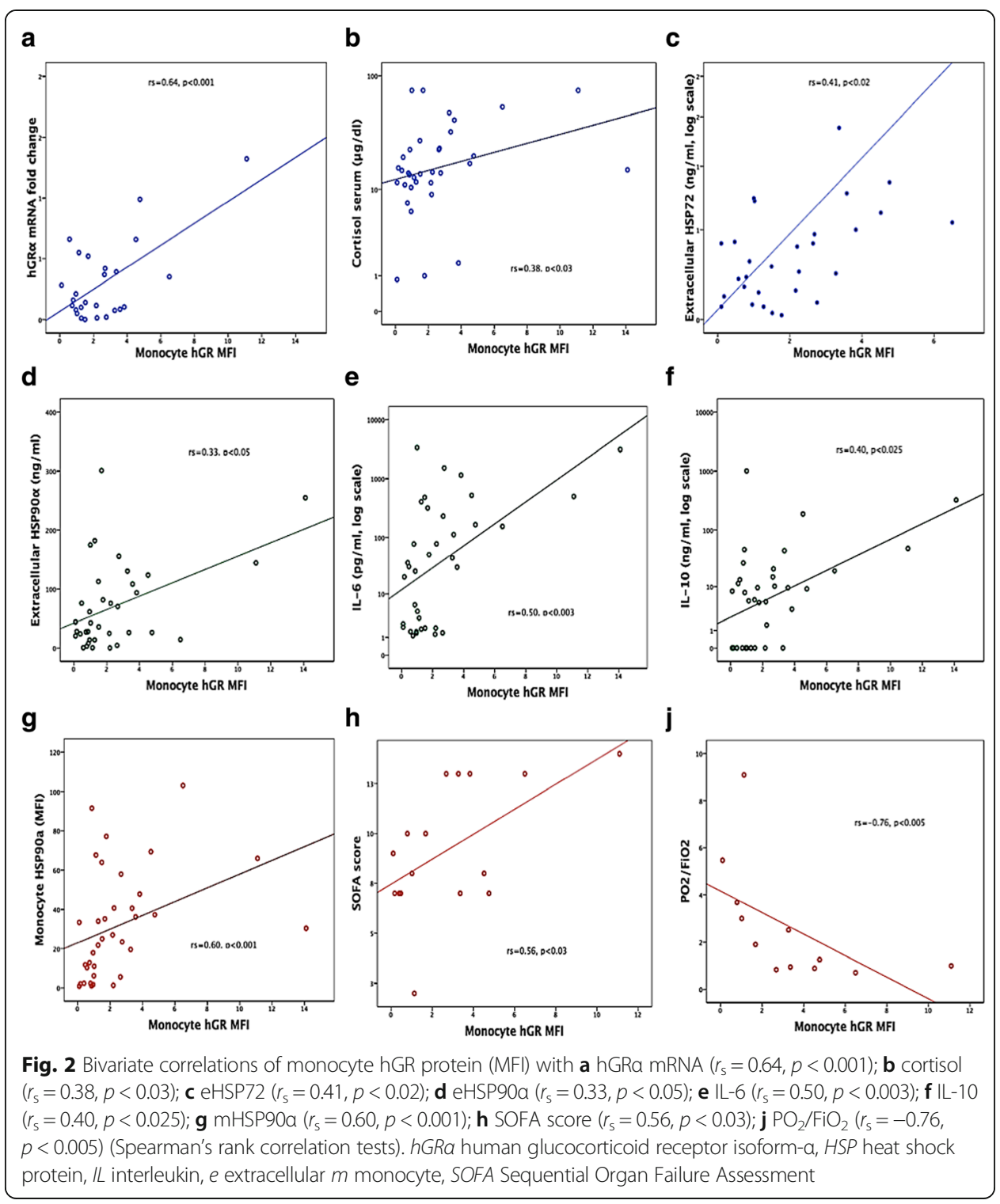

\section{Mortality}

More patients with sepsis died compared to those with SIRS (43.8 vs. $12.5 \%, p=0.001$ ) (Table 1). Within the sepsis group, more patients with septic shock showed a trend to die compared to those without (56 vs. $29 \%, p=0.08$ ). In sepsis group, APACHE II $(p<0.001)$, SAPS III $(p<0.02)$, hGR protein expression in monocytes $(p<0.04)$, and eHSP72 $(p<0.05)$ were higher among non-survivors compared to survivors. In SIRS, eHSP90 $\alpha$ levels $(p<0.02)$ were higher among non-survivors (Fig. 3a-f).

Regarding survivors, when septic and SIRS patients were compared, APACHE II $(p<0.03)$, SOFA $(p<0.03)$, SAPS III $(p<0.001)$, cortisol $(p=0.001)$, eHSP72 $(p<0.001)$, eHSP90 $\alpha(p<0.02)$, IL-6 $(p=0.005)$, and IL-10 $(p<0.01)$ were higher among septic compared to SIRS survivors. Regarding non-survivors, SAPS III $(p<0.01)$, cortisol $(p<0.02)$, ACTH $(p<0.04)$, eHSP72 $(p<0.05)$, and IFN- $\gamma(p<0.02)$ were higher among septic compared to SIRS patients (Fig. 3a-f). 


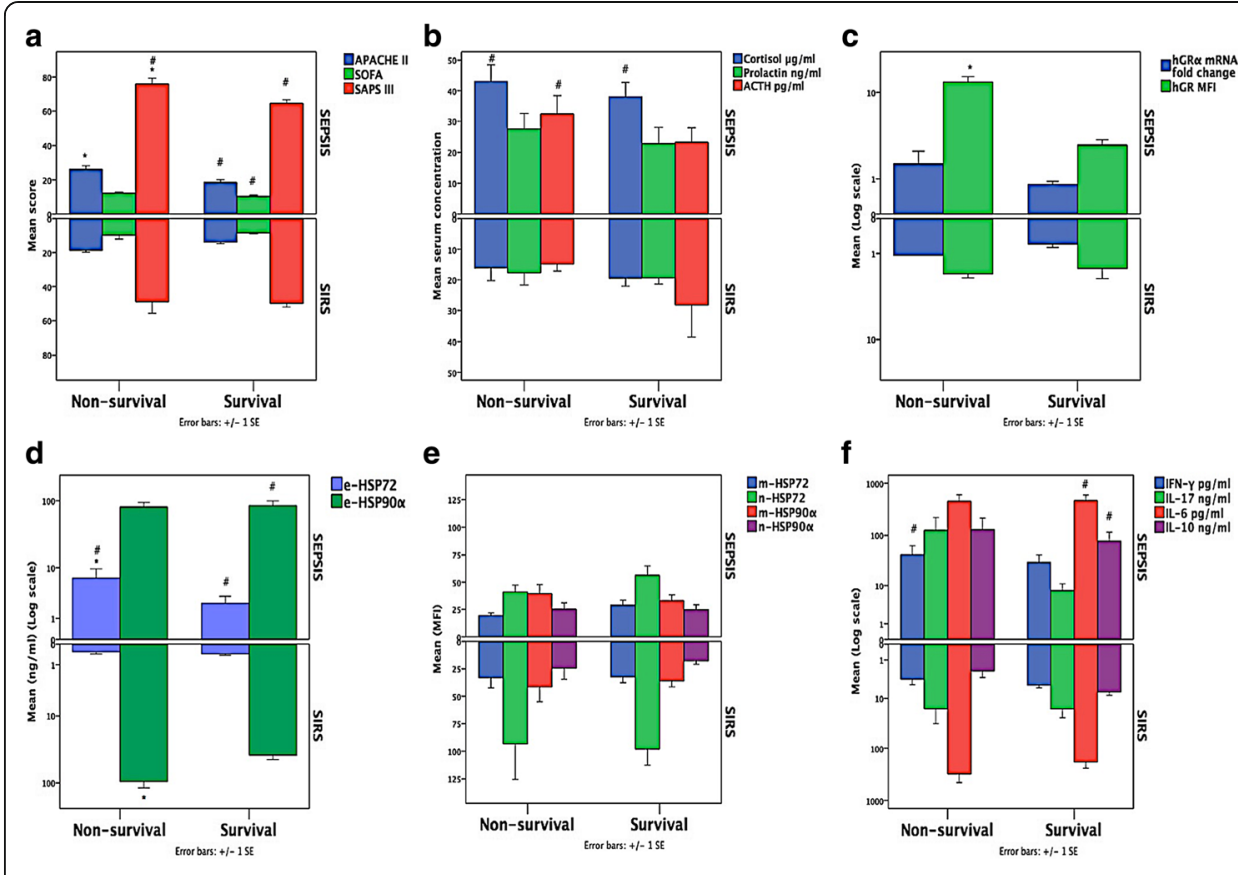

Fig. 3 Comparisons between survivors and non-survivors in the sepsis and SIRS subcohorts, regarding a severity of illness, b hormonal, c glucocorticoid receptor, $\mathbf{d}$ extracellular and e intracellular innate immune, and f inflammatory acute stress response. The $p$ value was calculated using the Mann-Whitney $U$ test and a $p$ value $<0.05$ (two sided) was considered statistically significant. Sepsis and/or mortality was associated with or had a trend for increased severity of illness, expression of extracellular HSP90a and HSP72, cytokines, cortisol, and GR and a trend for lower intracellular HSP72. Asterisk indicates $p<0.05$ between non-survivors and survivors in each diagnostic group; number sign indicates $p<0.05$ between sepsis and SIRS in each outcome group. SIRS systemic inflammatory response syndrome, APACHE // Acute Physiology and Chronic Evaluation, SOFA Sequential Organ Failure Assessment, SAPS III Simplified Acute Physiology Score III, GR glucocorticoid receptor, HSP heat shock protein, IL interleukin, IFN- $\gamma$ interferon gamma, MFI mean fluorescence intensity

Intracellular mHSP72 and nHSP72 were non-significantly decreased in sepsis compared to SIRS, especially among non-survivors. In the septic shock subcohort nHSP72 and nHSP90 $\alpha$ were more repressed compared to non-septic shock $(p<0.05)$, the hGR $\alpha$ mRNA showing a similar trend.

\section{hGR predictive values}

We generated ROC curves for hGR, stress-proteins (HSPs) expressing innate immunity, and cortisol with the best $p$ values for discriminating sepsis among ICU patients (Fig. 4a) or predicting mortality in the subpopulation of Sepsis-3 patients (Fig. 4b). Extracellular HSP72, cortisol, and hGR protein expression in monocyte best-discriminated sepsis from SIRS as indicated by an AUROC $>0.85$ with cutoff values and positive and negative predictive values presented in Table 2 . In the sepsis group only, monocyte-hGR protein expression and eHSP72 were strong predictors of mortality, achieving an AUROC > 0.95 with cutoff values and positive and negative predictive values presented in Table 3.

\section{Discussion}

In this study, we showed that early expression of hGR $\alpha$ mRNA or hGR protein expression in monocytes is significantly higher in patients with sepsis compared to healthy 


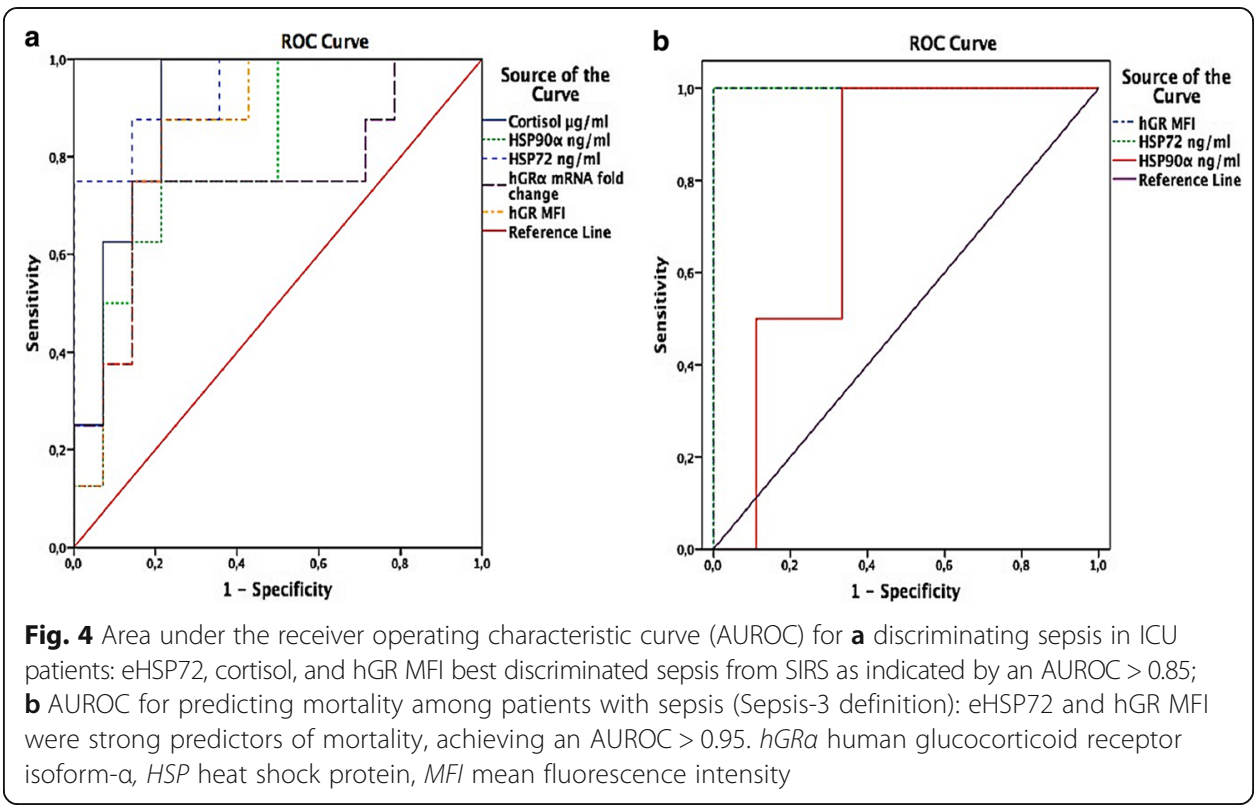

controls and/or patients with SIRS. Furthermore, we verified the hypothesis that the hormonal-innate immune stress-responding pairs of hGR-cortisol and hGR-HSPs are adequately expressed in the early phase of sepsis. We also showed that the hGR mRNA fold change and protein expression alterations are associated with simultaneous cortisol and extracellular or intracellular HSP72 or HSP90 $\alpha$ changes. It is possible that hGR is induced in monocytes, in association with the HSPs, to counter-balance the acute inflammatory response early in sepsis. We finally showed that in the acute phase of stress, hGR is able to predict sepsis and outcome and is related with stress-activated bio-molecules and organ dysfunction.

Our finding of upregulation of monocyte hGR expression could represent an adaptive response aiming at dampening the aggressive inflammation in early sepsis. This is in agreement with experimental studies showing increased hGR protein expression and binding capacity in circulating lymphocytes, monocytes, and splenocytes in murine endotoxic shock [18]. Earlier studies had shown lower hGR $\alpha$ mRNA trends in sepsis either in adult $\mathrm{T}$ lymphocytes [8] or in neutrophils from children [10] and decreased

Table 2 Receiver operating characteristic curve analysis to determine the optimum cutoff values and the positive and negative predictive values of hGR, cortisol, and HSPs for the prediction of sepsis in ICU patients

\begin{tabular}{|c|c|c|c|c|c|c|c|c|}
\hline \multirow[b]{2}{*}{ Biomarkers } & \multicolumn{8}{|l|}{ Parameters } \\
\hline & AUROC (95\% Cl) & $P$ value & Sensitivity & Specificity & J & Cutoff & PPV (\%) & NPV (\%) \\
\hline eHSP72 & $0.94(0.84-1.0)$ & 0.001 & 0.88 & 0.79 & 0.66 & 0.40 & 73 & 68 \\
\hline Cortisol & $0.90(0.77-1.0)$ & 0.002 & 1.00 & 0.79 & 0.79 & 15.7 & 66 & 70 \\
\hline hGR MFI & $0.85(0.69-1.0)$ & 0.008 & 0.88 & 0.79 & 0.66 & 2.66 & 73 & 49 \\
\hline eHSP90a & $0.80(0.62-0.99)$ & 0.02 & 0.75 & 0.79 & 0.54 & 66.0 & 72 & 56 \\
\hline hGRa mRNA & $0.75(0.52-0.98)$ & 0.05 & 0.75 & 0.86 & 0.61 & 0.38 & 66 & 67 \\
\hline
\end{tabular}

Abbreviations: $A U R O C$ area under the receiver operating characteristic curve, $C l$ confidence interval, $J$ Youden's index, PPV positive predictive value, NPV negative predictive value, eHSP extracellular heat shock protein, $h G R$ human glucocorticoid receptor, MFI mean fluorescent intensity 
Table 3 Receiver operating characteristic curve analysis to determine the optimum cutoff values and the positive and negative predictive values of hGR, cortisol, and HSPs for the prediction of mortality in ICU patients with Sepsis-3

\begin{tabular}{lllllllll}
\hline & Parameters \\
\cline { 2 - 8 } Biomarkers & AUROC (95\% Cl) & $P$ value & Sensitivity & Specificity & $J$ & Cutoff & PPV (\%) & NPV (\%) \\
\hline hGR MFI & $1.00(1.00-1.0) 0$ & 0.034 & 1.00 & 0.89 & 0.89 & 4.64 & 66 & 58 \\
eHSP72 & $1.00(1.00-1.00)$ & 0.034 & 1.00 & 0.89 & 0.89 & 2.1 & 53 & 61 \\
eHSP90a & $0.79(0.49-1.00)$ & 0.23 & 1.00 & 0.67 & 0.67 & 137.6 & 44 & 56 \\
\hline
\end{tabular}

Abbreviations: $A U R O C$ area under the receiver operating characteristic curve, $C l$ confidence interval, $J$ Youden's index, PPV positive predictive value, NPV negative predictive value, eHSP extracellular heat shock protein, $h G R$ human glucocorticoid receptor, MFI mean fluorescent intensity

${ }^{3} \mathrm{H}$-dexamethasone binding [19]. Importantly, total and cytoplasmic, but not nuclear, hGR protein levels were lower in PBMCs from small groups $(n=6-7)$ of children with sepsis or trauma compared to controls [20]. Earlier research was only done on mRNA, which in our study was shown to produce weaker response compared to protein. Downregulated hGR $\alpha$ in the liver, lung, and spleen but upregulated hGR $\alpha$ mRNA and binding activity have been reported in muscle in endotoxemic rats [21]. Inconsistency in selecting cell subpopulations or methods with different sensitivity in expressing or measuring hGR $\alpha$ might have produced such opposing results.

Although the anti-inflammatory GC actions have been suggested to be more effective in males than in females [22], we did not find any sex-specific influence on hGR $\alpha$ mRNA and hGR-protein expressions, or on serum cortisol, HSP, and ILs concentrations. Similar to our results, others have also observed that there is no correlation between hGR $\alpha$ mRNA levels and admission severity of illness [10]. By generating ROC curves for stress proteins and chaperones, we showed that hGR protein expression in monocytes, stress-proteins (HSPs) expressing innate immunity, and cortisol could discriminate sepsis among ICU patients with acceptable positive and negative predictive values. Acknowledging the limitation of the small sepsis-3 sample, high levels of hGR and eHSP72 were also able to predict mortality among septic patients. These chaperokines (HSPs), expressing innate-immunity, and targeted hormone-response receptors (hGR), seem to represent more sensitive biomarkers in predicting future events than simultaneously calculated or defined severity of illness scores and shock.

hGR $\alpha$ mRNA is produced much more slowly compared to corresponding hGR protein expression, translated at the same time [23]. This is because of several time-consuming steps at RNA processing not required for protein translation. Our finding, showing a good correlation of hGR protein in monocytes with hGR $\alpha$ mRNA, is consistent with reports in other human cells and tissues showing that hGR protein in most cells matches up well with hGR $\alpha$ mRNA levels [24]. Additionally, elevated hGR $\alpha$ mRNA levels correlate with the number of hormone binding sites in septic rats [25] and human lymphocytes [26], leading to increased hGR $\alpha$ binding activity and GC sensitivity [27]. Stimulation of T-cells upregulated the hGR $\alpha$, possibly rendering T-cells more sensitive to GC, although such a T-cell response was hindered by hydrocortisone [8]. Such a dynamic impact on the expression and function of hGR $\alpha$ might possibly explain why GC are beneficial only when administered early in sepsis [28]. Representing a tissue-specific adaptation, 
neutrophil hGR $\alpha$-binding capacity was decreased in murine sepsis [18] and neutrophil hGR $\alpha$ mRNA was decreased in septic children, leading to increased cortisol resistance of neutrophils, gradually normalized after recovery [10]. This is of particular importance since the prone to apoptosis during sepsis lymphocytes present opposing hGR $\alpha$ mRNA regulation from that of the prone to proliferation neutrophils. By adopting a whole-blood mRNA approach, we were able to provide a more comprehensive picture without missing hGR expression signatures from subpopulations that would potentially have been excluded from the experimental approach [29].

We showed for the first time that hGR protein and/or hGR $\alpha$ mRNA is associated with the cell chaperokines expressing innate immunity, extracellular and intracellular HSP72, and HSP90 $\alpha$, besides HPA, IL-6, IL-10, and IFN- $\gamma$. Expanding results of previous studies, showing an inverse hGR correlation to noradrenaline and lactate [28], we found that the hGR was also related with CRP, WBC, low $\mathrm{PO}_{2} / \mathrm{FiO} 2$, MODS, and SOFA scores. Although a negative correlation between hyperthermia and the number of receptors has been previously suggested [30], we could not find any relation between hGR $\alpha$ and temperature, lactate, glucose, LDH, IL-17, or PCT. In vitro, only heat shock induced mHSP72 and HSP72 mRNA but not lipopolysaccharide [31]. Our findings are in agreement with those of a recent study showing that in sepsis extracellular HSP72 and HSP90 $\alpha$ are increased, while intracellular HSPs are repressed [32]. These data probably imply a key role for the HSP72 and HSP90 $\alpha$ early in sepsis in converting the hGR $\alpha$ to the steroid-binding state [6]. The hGR $\alpha$-cortisol or ACTH relations and the HSP90 $\alpha$ time-regulation by the pattern of the pulsatile hormones, further support such a possibility [33].

The increased prolactin concentrations in our septic patients, have been previously shown to inhibit cellular immune functions in septic mice, decreasing survival [34], and to play a role in the acute stress response in sepsis [32]. Generation of cardiotoxic subfragments of prolactin caused by oxidative stress has been implicated in peripartum cardiomyopathy in women [35]. Although pharmacological blockade of prolactin might offer an innovative therapeutic intervention, the exact role of prolactin and its relation to hGR $\alpha$ in sepsis have still to be delineated.

The antibody and methodology used in our study allowed us to measure total hGR levels without distinction of isoforms, which constitutes a limitation of the current study. It is not possible to speculate if the higher hGR protein levels represent proportionally higher hGR $\beta$ levels, although we could not detect hGR $\beta$ on RNA level. The correlation of hGR protein with hGR $\alpha$ mRNA suggests that hGR is mainly representing $h G R \alpha$, reportedly expressed in extremely higher concentrations than hGR $\beta$ [10]. Weak correlations of hGR with some inflammatory, hormonal, or organ failure indices, although significant, should be interpreted with caution, probably attributed to the small sample numbers. Limitations of this study are also the lack of longitudinal data and the weakness of a single-center study. An intact HPA axis and GC response are critical to the host response to infectious agents. Also, infectious agents may directly modulate GR functioning [36]. The importance of GR $\alpha$ functioning is clearly shown in septic mice lacking endothelial GR $\alpha$ demonstrating increased mortality, hemodynamic instability, pro-inflammatory cytokine production [37], and inability to downregulate IL-1 $\beta$ [18]. Our finding of 
increased levels of hGR, cortisol, extracellular HSPs, and cytokines in sepsis, especially among non-survivors, probably reflects the intensity of the activation of the HPA axis and acute stress inflammatory and innate-immune response. This increase might not have been attributed to the cell death, not only since the increased hGR levels were unrelated to LDH levels but also because the simultaneously measured abundant intracellular HSP72 and 90 proteins were repressed rather than increased in the sepsis group and among non-survivors.

GCs may induce apoptosis by directly regulating typical apoptosis or survival genes or by inducing cellular distress that triggers the apoptotic cascade [38]. Results of this study indicate that the high-risk patient prepares himself to respond to the "danger" by increasing the eHSP72, eHSP90 $\alpha$, and cortisol levels in a "ready to bind to an enhanced hGR" state. The higher eHSP72 and lower nHSP72 we found in patients receiving hydrocortisone might probably indicate a higher degree of stress and inflammation in this group. The positive relations of the hGR to the inflammatory biomarkers further support this, along with the simultaneous enhanced ACTH and prolactin, and the organ dysfunction or failure development, when the cell defending itself represses its bioenergetics. Results of this study, showing that cortisol and hGR are increased in the acute phase of sepsis, might indicate that cortisol is not "a limiting factor" early in sepsis. Thus, exogenous cortisol early in sepsis might repress the already enhanced hGR expression through a negative feedback mechanism, increasing the GC resistance. Although an increased hGR expression has also been shown in T lymphocytes during septic shock, regardless of GC treatment, hGR binding capacity was decreased in neutrophils in GC-treated patients [28]. We did not find any monocyte or whole blood hGR difference between patients started on stress hydrocortisone and those not receiving GCs. The decreased neutrophil capacity to bind hGR $\alpha$ in sepsis, however, might impede the response to GC treatment. Accordingly, the use of GCs in septic shock may be dependent on the stage of the sepsis, the reactivity of the HPA axis, and the sensitivity of hGR to the ligand [36]. Non-steroidal selective hGR modulators (Sin EGRAMs) that can activate specific hGR mechanisms and thus alter hGR $\alpha$-mediated gene expression profiles have now gained more interest using mutant hGR research [39].

\section{Conclusions}

In conclusion, early expression of hGR $\alpha$ mRNA or hGR $\alpha$ protein is significantly higher in patients with sepsis compared to healthy controls and/or patients with SIRS. Early in critical illness, the hGR expression can predict sepsis and outcome and is related with stress-activated bio-molecules and organ dysfunction. It is possible that the hGR, in association with the HSPs, is induced in monocytes to facilitate the cortisol binding to counter-balance the increased inflammatory response to the acute stress in early sepsis. Exogenous cortisol in the acute phase of sepsis might decrease the hGR expression through a negative feedback mechanism, increasing the GC resistance. Hopefully, smart research might in future lead to a more individualized approach to GC treatment in sepsis and septic shock, taking into account both the hGR $\alpha$ expression and binding capacity, as well as the balance of the immune-hormonal response. 


\section{Publication of clinical datasets}

Our dataset contains clinical data, for which we had undertaken an ethical and legal responsibility to respect participants' rights to privacy and to protect their identity. The already received informed consents from participants at the point of recruitment to the trial did not mention any consent for publishing raw data. The institutional review board of Evangelismos Hospital had also demanded all data to be kept privately and not to be distributed. We should have known this possibility to include in our initial project and consent, which, however, we did not know. It is impossible now for the dataset to be considered for publication, because we may compromise anonymity or confidentiality or breach local data protection laws regarding patients' and healthy individuals' data. Patients and controls in an institution in a small period are well known, and it is now impossible to obtain a retrospective consent regarding publication of data.

\footnotetext{
Abbreviations

AP-1: Activator protein-1; APACHE II: Acute Physiology and Chronic Evaluation; DBD: DNA-binding domain; GCs: Glucocorticoids; GRE: Glucocorticoid response elements; hGR: Human glucocorticoid receptor; HPA: Hypothalamic-pituitary-adrenal; HSP: Heat shock protein; ICU: Intensive care unit; ILs: Interleukins; LBD: Ligandbinding domain; LOS: Length of stay; NF-kB: Nuclear factor-kB; NLS: Nuclear localization sequences; RT-PCR: Real-time PCR; SAPS III: Simplified Acute Physiology Score III; SIRS: Systemic inflammatory response syndrome; SOFA: Sequential Organ Failure Assessment; STATs: Signal transducers and activators of transcription
}

\section{Acknowledgements}

This research has been co-financed by the European Union (European Social Fund (ESF)) and Greek national funds through the Operational Program "Education and Lifelong Learning" of the National Strategic Reference Framework (NSRF)-Research Funding Program: THALES.

\section{Competing interests}

The authors declare that they have no competing interests.

\section{Authors' contributions}

KV and KA have generated, collected, and assembled the data; GB, EC, and CAS conceived and designed the study; AS, KP, DG, EBR, EBO, and ST substantially contributed to the collection and analysis of samples; SI, CR, and EM have substantially contributed to the interpretation of data and revision and editing of the manuscript; KV, SN, and GB organized patient recruitment, analyzed and interpreted the data, and wrote the manuscript; all authors edited and approved the final version of the manuscript.

\section{Author details \\ ${ }^{1}$ First Critical Care Department, National and Kapodistrian University of Athens, Athens, Greece. ${ }^{2}$ Pediatric Intensive Care Unit, University Hospital, University of Crete, 71500 Heraklion, Crete, Greece. 'Division of Endocrinology, Metabolism and Diabetes, First Department of Pediatrics, 'Aghia Sophia' Children's Hospital and Division of Endocrinology and Metabolism, Biomedical Research Foundation of the Academy of Athens, National and Kapodistrian University of Athens, Athens, Greece. ${ }^{4}$ First Department of Internal Medicine-Propaedeutic, National and Kapodistrian University of Athens, Athens, Greece. ${ }^{5}$ Immunology-Histocompatibility Department, Evangelismos Hospital, Athens, Greece. ${ }^{6}$ Department of Endocrinology-Diabetes, Evangelismos Hospital, Athens, Greece. ${ }^{7}$ Section on Endocrinology and Genetics, Eunice Kennedy Shriver National Institute of Child Health and Human Development (NICHD), National Institutes of Health, Bethesda, MD 20892, USA.}

Received: 28 December 2016 Accepted: 9 February 2017

Published online: 21 February 2017

\section{References}

1. Singer M, Deutschman CS, Seymour CW et al (2016) The Third International Consensus Definitions for Sepsis and Septic Shock (Sepsis-3). JAMA 315:801-810. doi:10.1001/jama.2016.0287

2. Peeters RP, Hagendorf A, Vanhorebeek I et al (2009) Tissue mRNA expression of the glucocorticoid receptor and its splice variants in fatal critical illness. Clin Endocrinol (Oxf) 71:145-153. doi:10.1111/j.1365-2265.2008.03443.x

3. Boonen E, Van den Berghe G (2014) Cortisol metabolism in critical illness: implications for clinical care. Curr Opin Endocrinol Diabetes Obes 21:185-192. doi:10.1097/MED.0000000000000066

4. Nicolaides NC, Galata Z, Kino T et al (2010) The human glucocorticoid receptor: molecular basis of biologic function. Steroids 75:1-12. doi:10.1016/j.steroids.2009.09.002

5. Oakley RH, Jewell CM, Yudt MR et al (1999) The dominant negative activity of the human glucocorticoid receptor beta isoform. Specificity and mechanisms of action. J Biol Chem 274:27857-27866

6. Kirschke E, Goswami D, Southworth D et al (2014) Glucocorticoid receptor function regulated by coordinated action of the Hsp90 and Hsp70 chaperone cycles. Cell 157:1685-1697. doi:10.1016/j.cell.2014.04.038 
7. Okret S, Dong Y, Brönnegård M, Gustafsson JA (1991) Regulation of glucocorticoid receptor expression. Biochimie 73:51-59

8. Ledderose C, Möhnle P, Limbeck E et al (2012) Corticosteroid resistance in sepsis is influenced by microRNA-124-induced downregulation of glucocorticoid receptor-a. Crit Care Med 40:2745-2753. doi:10.1097/CCM.0b013e31825b8ebc

9. Guerrero J, Gatica HA, Rodríguez M et al (2013) Septic serum induces glucocorticoid resistance and modifies the expression of glucocorticoid isoforms receptors: a prospective cohort study and in vitro experimental assay. Crit Care Lond Engl 17:R107. doi:10.1186/cc12774

10. van den Akker ELT, Koper JW, Joosten K et al (2009) Glucocorticoid receptor mRNA levels are selectively decreased in neutrophils of children with sepsis. Intensive Care Med 35:1247-1254. doi:10.1007/s00134-009-1468-6

11. Vincent JL, Moreno R, Takala J et al (1996) The SOFA (Sepsis-related Organ Failure Assessment) score to describe organ dysfunction/failure. On behalf of the Working Group on Sepsis-Related Problems of the European Society of Intensive Care Medicine. Intensive Care Med 22:707-710

12. Shankar-Hari M, Phillips GS, Levy ML et al (2016) Developing a new definition and assessing new clinical criteria for septic shock: for the Third International Consensus Definitions for Sepsis and Septic Shock (Sepsis-3). JAMA 315:775-787. doi:10.1001/jama.2016.0289

13. Kaukonen K-M, Bailey M, Pilcher D et al (2015) Systemic inflammatory response syndrome criteria in defining severe sepsis. N Engl J Med 372:1629-1638. doi:10.1056/NEJMoa1415236

14. Knaus WA, Draper EA, Wagner DP, Zimmerman JE (1985) APACHE II: a severity of disease classification system. Crit Care Med 13:818-829

15. Marshall JC, Cook DJ, Christou NV et al (1995) Multiple organ dysfunction score: a reliable descriptor of a complex clinical outcome. Crit Care Med 23:1638-1652

16. Moreno RP, Metnitz PGH, Metnitz B et al (2008) Modeling in-hospital patient survival during the first 28 days after intensive care unit admission: a prognostic model for clinical trials in general critically ill patients. J Crit Care 23: 339-348. doi:10.1016/j.jcrc.2007.11.004

17. Charmandari E, Chrousos GP, Lambrou Gl et al (2011) Peripheral CLOCK regulates target-tissue glucocorticoid receptor transcriptional activity in a circadian fashion in man. PLoS One 6, e25612. doi:10.1371/journal.pone.0025612

18. Bergquist $M$, Jirholt $P$, Nurkkala $M$ et al (2014) Glucocorticoid receptor function is decreased in neutrophils during endotoxic shock. J Infect 69:113-122. doi:10.1016/j.jinf.2014.03.011

19. Siebig S, Meinel A, Rogler G et al (2010) Decreased cytosolic glucocorticoid receptor levels in critically ill patients. Anaesth Intensive Care 38:133-140

20. Indyk JA, Candido-Vitto C, Wolf IM et al (2013) Reduced glucocorticoid receptor protein expression in children with critical illness. Horm Res Pædiatrics 79:169-178. doi:10.1159/000348290

21. Duma D, Silva-Santos JE, Assreuy J (2004) Inhibition of glucocorticoid receptor binding by nitric oxide in endotoxemic rats. Crit Care Med 32:2304-2310

22. Duma D, Collins JB, Chou JW, Cidlowski JA (2010) Sexually dimorphic actions of glucocorticoids provide a link to inflammatory diseases with gender differences in prevalence. Sci Signal 3:ra74. doi:10.1126/scisignal.2001077

23. Vogel C, Marcotte EM (2012) Insights into the regulation of protein abundance from proteomic and transcriptomic analyses. Nat Rev Genet 13:227-232. doi:10.1038/nrg3185

24. Pujols L, Mullol J, Roca-Ferrer J et al (2002) Expression of glucocorticoid receptor alpha- and beta-isoforms in human cells and tissues. Am J Physiol Cell Physiol 283:C1324-C1331. doi:10.1152/ajpcell.00363.2001

25. Sun X, Fischer DR, Pritts TA et al (2002) Expression and binding activity of the glucocorticoid receptor are upregulated in septic muscle. Am J Physiol Regul Integr Comp Physiol 282:R509-R518. doi:10.1152/ajpregu.00509.2001

26. Goecke IA, Alvarez C, Henríquez I et al (2007) Methotrexate regulates the expression of glucocorticoid receptor alpha and beta isoforms in normal human peripheral mononuclear cells and human lymphocyte cell lines in vitro. Mol Immunol 44:2115-2123. doi:10.1016/j.molimm.2006.07.303

27. Molijn GJ, Spek JJ, van Uffelen JC et al (1995) Differential adaptation of glucocorticoid sensitivity of peripheral blood mononuclear leukocytes in patients with sepsis or septic shock. J Clin Endocrinol Metab 80:1799-1803. doi:10.1210/jcem.80.6.7775626

28. Bergquist M, Lindholm C, Strinnholm M et al (2015) Impairment of neutrophilic glucocorticoid receptor function in patients treated with steroids for septic shock. Intensive Care Med Exp 3:59. doi:10.1186/s40635-015-0059-9

29. Wong HR (2012) Clinical review: sepsis and septic shock-the potential of gene arrays. Crit Care Lond Engl 16:204. doi:10.1186/cc10537

30. Molijn GJ, Koper JW, van Uffelen CJ et al (1995) Temperature-induced down-regulation of the glucocorticoid receptor in peripheral blood mononuclear leucocyte in patients with sepsis or septic shock. Clin Endocrinol (Oxf) 43:197-203

31. Briassouli E, Tzanoudaki M, Goukos D et al (2015) Glutamine may repress the weak LPS and enhance the strong heat shock induction of monocyte and lymphocyte HSP72 proteins but may not modulate the HSP72 mRNA in patients with sepsis or trauma. BioMed Res Int 2015:806042. doi:10.1155/2015/806042

32. Vardas K, Apostolou K, Briassouli E et al (2014) Early response roles for prolactin cortisol and circulating and cellular levels of heat shock proteins 72 and 90a in severe sepsis and SIRS. BioMed Res Int 2014:803561. doi:10. $1155 / 2014 / 803561$

33. Conway-Campbell BL, George CL, Pooley JR et al (2011) The HSP90 molecular chaperone cycle regulates cyclical transcriptional dynamics of the glucocorticoid receptor and its coregulatory molecules CBP/p300 during ultradian ligand treatment. Mol Endocrinol Baltim Md 25:944-954. doi:10.1210/me.2010-0073

34. Oberbeck R, Schmitz D, Wilsenack K et al (2003) Prolactin modulates survival and cellular immune functions in septic mice. J Surg Res 113:248-256

35. Sliwa K, Hilfiker-Kleiner D, Petrie MC et al (2010) Current state of knowledge on aetiology, diagnosis, management, and therapy of peripartum cardiomyopathy: a position statement from the Heart Failure Association of the European Society of Cardiology Working Group on peripartum cardiomyopathy. Eur J Heart Fail 12:767-778. doi:10.1093/eurjhf/hfq120

36. Webster Jl, Sternberg EM (2004) Role of the hypothalamic-pituitary-adrenal axis, glucocorticoids and glucocorticoid receptors in toxic sequelae of exposure to bacterial and viral products. J Endocrinol 181:207-221 
37. Goodwin JE, Feng Y, Velazquez H, Sessa WC (2013) Endothelial glucocorticoid receptor is required for protection against sepsis. Proc Natl Acad Sci U S A 110:306-311. doi:10.1073/pnas.1210200110

38. Schmidt S, Rainer J, Ploner C et al (2004) Glucocorticoid-induced apoptosis and glucocorticoid resistance: molecular mechanisms and clinical relevance. Cell Death Differ 11(Suppl 1):S45-S55. doi:10.1038/sj.cdd.4401456

39. Sundahl N, Bridelance J, Libert C et al (2015) Selective glucocorticoid receptor modulation: new directions with non-steroidal scaffolds. Pharmacol Ther 152:28-41. doi:10.1016/j.pharmthera.2015.05.001

Submit your manuscript to a SpringerOpen ${ }^{\circ}$ journal and benefit from:

- Convenient online submission

- Rigorous peer review

- Immediate publication on acceptance

- Open access: articles freely available online

- High visibility within the field

- Retaining the copyright to your article

Submit your next manuscript at $>$ springeropen.com 